\title{
Gefängnisärztin
}

\section{Tanja Kühnle}

Dipl.-Biol., Managing Editor

Die letzte Prüfung ist geschafft, das Medizinstudium beendet. Und wie geht es jetzt weiter? In unserer Serie «Du findest Deinen Weg!» stellen wir Ihnen in unregelmässigen Abständen aussergewöhnliche Berufsperspektiven für Mediziner vor. In dieser Ausgabe berichtet Dr. med. Bidisha Chatterjee von ihrer Tätigkeit als Gefängnisärztin.*

\section{Frau Chatterjee, wie kam es, dass Sie Gefängnisärztin} geworden sind?

Ich war als Oberärztin in der Klinik für Allgemeine Innere Medizin im Inselspital Bern angestellt und mein Chefarzt hat mich als Anstaltsärztin in der Frauenanstalt in Hindelbank eingeteilt. Zusätzlich hat er mir die Abteilung im Inselspital für Gefängnisinsassen übertragen. Zuerst wollte ich kündigen. Aber dann habe ich mir gedacht, dass ich mir das auch mal anschauen kann - und habe einen spannenden Bereich vorgefunden: engagierte Pflegefachfrauen, die vor Ort den Gesundheitsdienst managen und im Vergleich zum Spital mit vielen schwierigen Situationen konfrontiert sind. Und viele jüngere Patienten, die teilweise sehr wenig mit medizinischer Versorgung in Kontakt gekommen sind und die man mit einfachen Massnahmen behandeln kann.

\section{Benötigen Sie für diesen Job eine spezielle Zusatz- ausbildung?}

Nein, es ist allerdings hilfreich, wenn man in den Gebieten Sucht/Substitution und Psychiatrie/Psychosomatik Bescheid weiss. Ebenso ist es von Vorteil, wenn man die Abläufe im Gefängnis ein bisschen kennt. Und es hilft, wenn man verschiedene Sprachen spricht und sich für die Personen aus anderen Kulturen interessiert.

\section{Wie viele Sprachen sprechen Sie?}

Ich spreche Deutsch, Französisch, Englisch, Italienisch, Spanisch und ein bisschen Arabisch. Damit kann man schon einen recht grossen Bereich abdecken. Aber wenn jemand beispielsweise Georgisch spricht, dann kann ich auch einen Übersetzer hinzuziehen. Das geht am einfachsten über den Dolmetscherdienst, den man jederzeit per Telefon erreichen und zuschalten kann.

\section{Sind Sie ausschliesslich als Gefängnisärztin tätig?}

Nein. Bis Ende August war ich Amtsärztin beim Amt für Justizvollzug und sowohl für das Gefängnis in

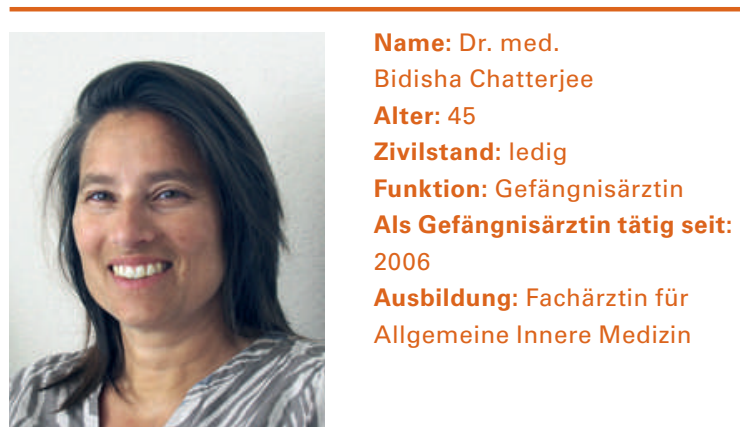

Burgdorf wie auch für das Jugendheim Lory und bis letzten September für die Anstalten Hindelbank zuständig. Derzeit arbeite ich noch als Projektleiterin beim Amt für Justizvollzug weiter. Zudem bin ich noch für das Untersuchungsgefängnis Solothurn tätig und werde nun den Einstieg in eine Hausarztpraxis angehen.

\section{Dürfen Sie als Frau nur in einem Frauengefängnis als Ärztin arbeiten?}

Nein, da gibt es keine Einschränkungen. Ich darf in jedem Gefängnis als Ärztin tätig sein. Im Untersuchungsgefängnis Solothurn sind beispielsweise Frauen und Männer inhaftiert.

\section{Wie sieht ein typischer Arbeitstag aus?}

Am Morgen habe ich meistens Sprechstunde. Der Gesundheitsdienst vor Ort hat bereits alle Patienten, die zum Arzt gehen wollen, angeschaut und eine Sprechstundenliste zusammengestellt. Ich sehe die Patienten nicht sehr regelmässig und nehme mir dann in der Sprechstunde Zeit für sie. Anschliessend folgen Besprechungen und die Dokumentation. Am Nachmittag bin ich dann meistens mit übergeordneten Aufgaben für das Amt für Justizvollzug beschäftigt.

\section{Können Sie ein Beispiel nennen?}

Momentan beschäftige ich mich mit Projekten wie dem elektronischen Patientendossier. Ausserdem nehme ich 


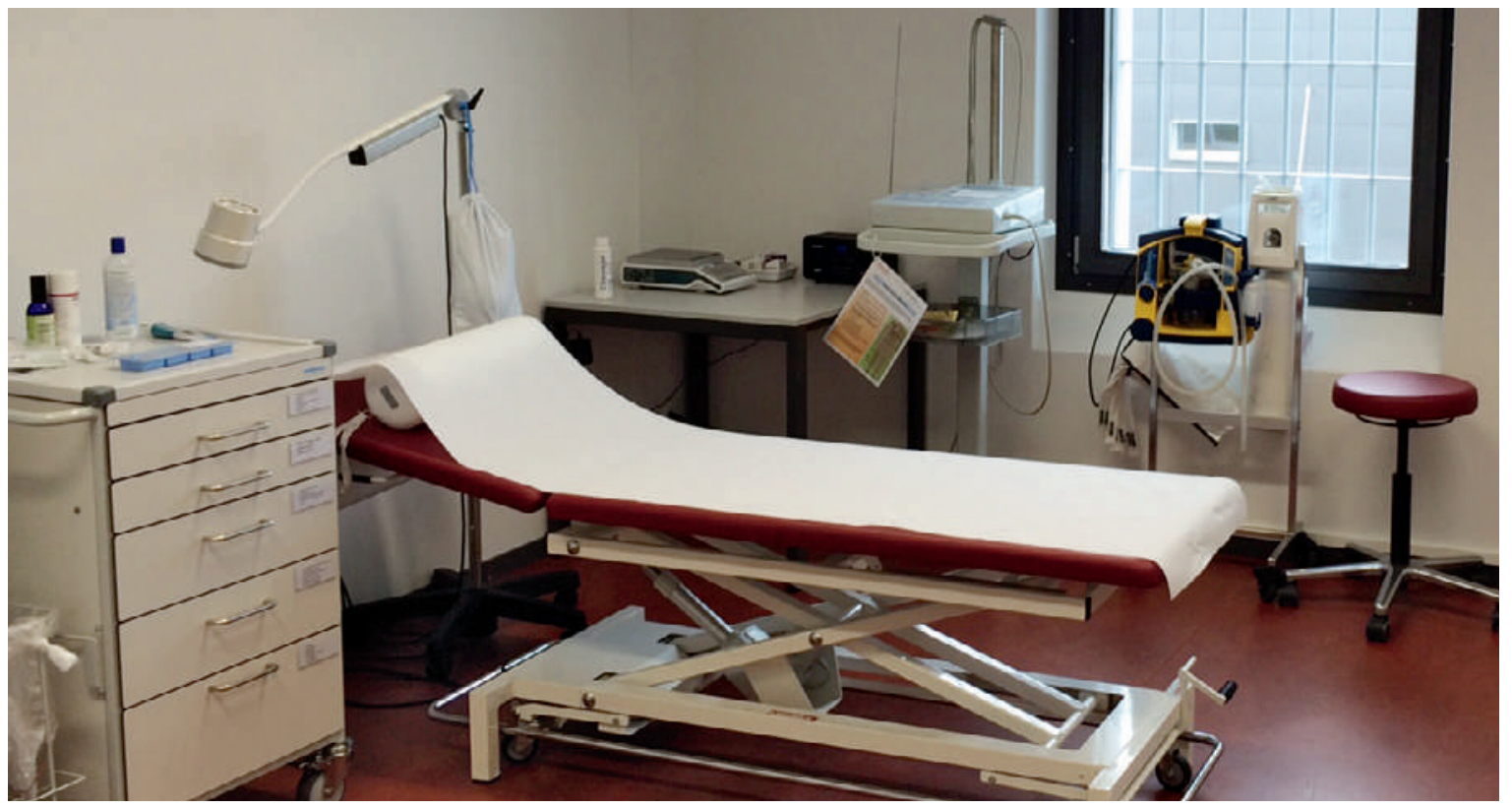

Der Behandlungsraum im Regionalgefängnis Burgdorf. (Foto: Bidisha Chatterjee)

meine Aufgaben als Präsidentin von Sante Prison Suisse wahr, dem schweizerischen interdisziplinären Fachrat für Gesundheitsfragen im Justizvollzug. In den zehn Jahren, in denen ich als Gefängnisärztin tätig bin, bin ich zur Fachspezialistin geworden. Ich werde deshalb häufig als Expertin beigezogen, beispielsweise vom Staatssekretariat für Migration, der Polizei, den Fachgruppen Sucht, anderen Gefängnissen und Anstalten in der Schweiz, aber auch von internationalen Delegationen.

\section{Welche Erkrankungen sind im Gefängnis typisch?}

Schlafprobleme und damit verbunden: Nackenschmerzen, Rückenschmerzen, Kopfschmerzen, Schwindel und Obstipation. Zahnprobleme sind auch sehr häufig.

\section{"Gefängnis" und "Anstalt» - wie unterscheiden sie sich?}

Das Gefängnis wird auch Untersuchungsgefängnis (UG), Regionalgefängnis (RG) oder Bezirksgefängnis genannt. Die Insassen befinden sich meist in Untersuchungshaft. Nach einer Festnahme durch die Polizei muss innerhalb von 24 Stunden ein Haftrichter über die Notwendigkeit einer Untersuchungshaft entscheiden. Meistens wird diese im Zusammenhang mit Fluchtgefahr, Verdacht auf weiterbestehende Gefahr für Delikte, Kollusionsgefahr mit anderen Personen ausgesprochen. Die Dauer beträgt meist drei Monate. In dieser Zeit ist der Alltag sehr eintönig: 23 Stunden in der Zelle, eine Stunde Spazierhof, manchmal ein bisschen Arbeit, wenig Besuche. Je nach Ermittlungsstand werden die Personen zu Einvernahmen, Tatortbegehungen oder Gegenüberstellungen etc. abgeholt. Aufgrund von Platzknappheit befinden sich in einem Untersuchungsgefängnis auch Personen, die nur kurze Strafen von bis zu drei Monaten zu verbüssen haben sowie Personen in Ausschaffungshaft. Manchmal auch Personen mit langen Strafen, die umplatziert werden müssen.

Ist eine Person verurteilt, wird sie zur Verbüssung der Strafe in eine Anstalt verlegt. Dort erfolgt die Unterbringung meist in Wohngruppen. Die Regelungen bezüglich Telefonaten, Besuchen, Sport und Bildung sind freier, es besteht eine Arbeitspflicht. Während des Aufenthalts in der Anstalt werden weitreichende Vorbereitungen für die Zeit nach der Haftstrafe getroffen.

\section{Haben Frauen andere gesundheitliche Probleme} als Männer?

Ja, Frauen sind mehr mit dermatologischen und ernährungstechnischen Problemen beschäftigt - Haarausfall und Gewichtszunahmen werden häufig beklagt.

\section{Kommt es vor, dass ein Häftling eine Erkrankung simuliert? Und wenn ja, warum?}

Simulieren würde ich das nicht nennen. Ich glaube, es gibt viele Beschwerden, die in den psychosomatischen Bereich fallen. Die Personen haben viel Zeit, sich mit sich selber zu beschäftigen. Die Ernährung ist anders, die Bewegungsfreiheit eingeschränkt, man ist nicht viel an der frischen Luft, man hat häufig keine Beschäftigung. Da fällt einem schon mal ein Zwicken und Stechen auf oder ein merkwürdiger Hautfleck, den man bislang nicht beachtet hat.

\section{Fällt es Ihnen schwer, jemanden zu behandeln, der} einen anderen Menschen getötet hat?

Nein, meine Aufgabe ist, das medizinische Problem zu beheben. Ich bin keine Richterin.

\section{Einige Insassen kennen Sie seit Jahren. Ziehen die Häftlinge Sie manchmal ins Vertrauen?}

Ja, ich höre auch zu, erkläre aber auch immer, was meine Aufgaben sind und was nicht. Ich bin keine Mitarbeiterin der Behörde, die Fragen zu Telefonaten und Berichten klärt.

Sind Sie mit dem Häftling allein, wenn Sie ihn behandeln? Nein, es ist eine Pflegefachfrau vor Ort. 


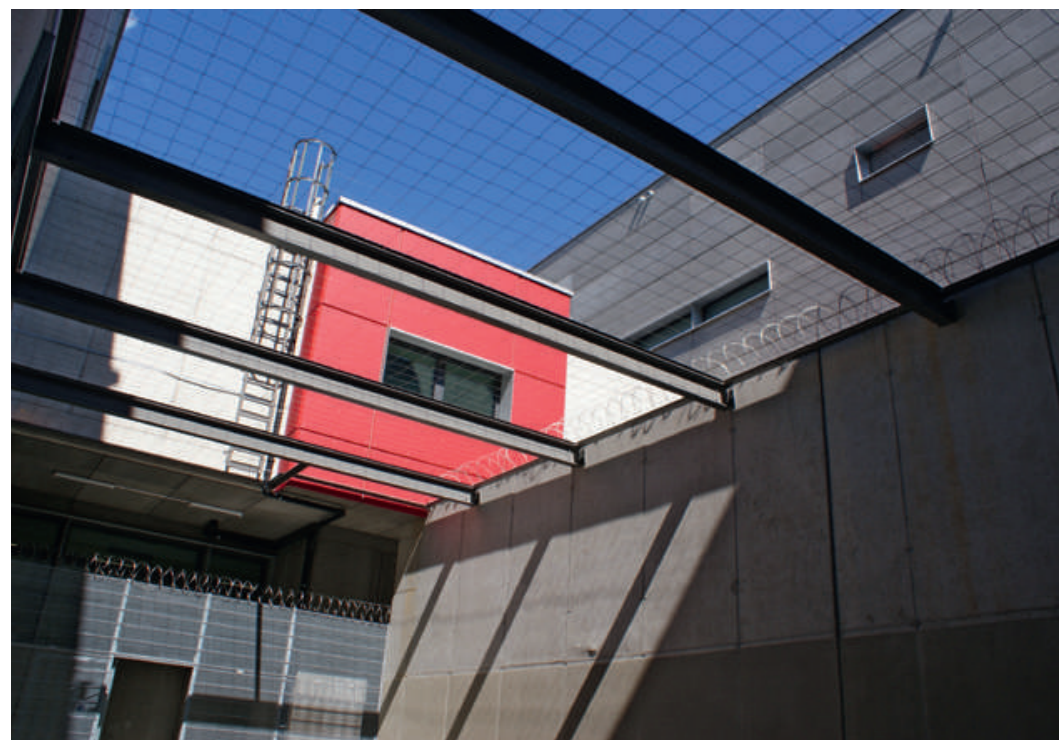

Blick aus dem Innenhof des Regionalgefängnisses in Burgdorf. (Foto: RG Burgdorf)

Wenn Sie eine Platzwunde nähen, hantieren Sie mit Schere, Skalpell und Nadel. Herrscht dann eine erhöhte Sicherheitsstufe?

Nein.

\section{Haben Sie manchmal Angst, dass Ihnen ein Häftling etwas antut?}

Nein. Ich signalisiere auch, dass man mich nicht anfluchen und beleidigen muss, denn sonst mache ich keine Behandlung. Ohnehin sind die Patienten eigentlich immer froh, wenn sie zum Arzt kommen können. Und zudem habe ich die Erfahrung gemacht: Ernstnehmen und Zuhören beruhigt viele Situationen.

\section{Waren Sie denn schon einmal in einer gefährlichen Situation? \\ Nein.}

\section{Hätten Sie denn im Ernstfall die Möglichkeit, Hilfe zu} holen?

Ja, es gibt einen Alarmknopf. Aber ich habe ihn bislang noch nie gebrauchen müssen.

\section{Nehmen wir an, ein Häftling erleidet in seiner Zelle plötzlich einen Herzinfarkt. Wie erfahren Sie davon und was passiert dann?}

Jeder Häftling kann sich über einen Zellenruf bemerkbar machen. Wir rufen dann die Ambulanz und der Patient kommt ins Spital. Und wenn uns bei einem Insassen bekannt ist, dass er zum Beispiel an Epilepsie leidet, dann bringen wir ihn nur gemeinsam mit anderen Gefangenen unter, damit diese uns im Ernstfall alarmieren können.
Endet Ihre Zuständigkeit, wenn ein Insasse entlassen oder verlegt wird?

An sich ja. Aber ich versuche immer, den Insassen für den Übergang so gut es geht zu versorgen. Zum Beispiel, wenn der Insasse an einem Methadonprogramm teilnimmt und nicht klar ist, ob er nach dem Ortswechsel sofort wieder Zugang zum Medikament bekommt. Ich versuche dann mit dem Arzt vor Ort Kontakt aufzuneh men. Das ist manchmal gar nicht so einfach, beispielsweise wenn der Insasse in sein Heimatland zurückkehrt.

\section{Lässt sich Ihr Beruf gut mit der Familie vereinbaren?} $\mathrm{Ja}$, genauso wie alle anderen praktischen Bereiche des «Arztlebens».

\section{Was fasziniert Sie an Ihrem Beruf am meisten?}

Ich kann nicht beeinflussen, wieso und wie lange jemand im Gefängnis sein muss. Ich erkenne aber, dass häufig das Delikt die Folge einer Verkettung von ungünstigen Situationen und Ereignissen ist. Mitzuhelfen, dass die Strafe unter humanen Bedingungen und mit einer professionellen medizinischen Versorgung erfolgt, ist für mich ein hohes Ziel.

\section{Was war Ihre bislang grösste Herausforderung als Gefängnisärztin?}

Ich kann mich an keine spezielle Situation erinnern. Aber was häufig schwierig ist: der Spagat zwischen Medizin und Behörde. Im Spital muss man nie erklären, wieso man eine Behandlung macht. Im Gefängnis immer. Häufig kriegt man zu hören: «Da wird jedes Wehwehchen behandelt. Die jammern nur und haben nichts.» Ebenso wird immer wieder kritisiert, dass man einen Extraaufwand für einen Patienten tätigen muss, weil der Arzt das so will. Das ist anstrengend und zermürbend. Eine Herausforderung sind auch die zunehmenden Kostenprobleme. Denn viele Personen sind nicht versichert, die Kantone müssen sparen. Und mehr und mehr Personen sind ohne Aufenthaltsstatus in der Schweiz im Gefängnis. Das macht die Situation nicht einfacher.

\section{Ihr Ratschlag für junge Kollegen, die auch Gefängnis-} arzt werden wollen:

Sich für die medizinischen Probleme der Patienten zu interessieren. Nicht allzu sehr auf die Delikte zu fokussieren, sondern einfach den Menschen zuzuhören und dann aufgrund der Beschwerden die bestmögliche Therapie auszuwählen.

Haben Sie als Mediziner auch einen aussergewöhnlichen Beruf, den Sie unseren Lesern gern vorstellen möchten? Dann freuen wir uns auf Ihre Bewerbung per E-Mail an: tkuehnle[at]emh.ch 